\title{
PHOTOINDUCED CHARGE SEPARATION PROCESSES IN SUPRAMOLECULAR TRIAD SYSTEMS*
}

\author{
K. Pirowska and J. NaJBaR ${ }^{\dagger}$ \\ Department of Physical Chemistry and Electrochemistry \\ Jagiellonian University, Ingardena 3, 30-060 Kraków, Poland \\ (Received July 23, 1998)
}

The nonadiabatic electron transfers in donor-acceptor-acceptor systems are investigated using three potential energy surfaces and two reaction coordinates via the stochastic Liouville equation to describe time evolution of the three excited electronic states: (1) $D^{*}-A-A$, (2) $D^{+}-A^{-}-A$ and (3) $D^{+}-A-A^{-}$. The electronic dephasing processes are taken into account phenomenologically in terms of dephasing constants. The couplings between surfaces are effective along the intersections of pairs of surfaces in the two-dimensional coordinate space. Special situations occur in the reaction coordinate space when three surfaces are nearly degenerate. The interplay between the sequential electron transfer processes and the superexchange process is analysed for different: reorganization energies, electronic coupling, free energies for the electron transfer, dephasing rates, and temperature. The time dependent contribution of the superexchange process to the charge separation in the triad system is analysed using the time dependent rate functions. It is shown that in the nonadiabatic limit of electron transfer the influence of the electronic dephasing processes for low barrier reactions can be accounted for by appropriate changes in the reorganization energies. The present model is compared with the experimental results concerning the charge separation in the bacterial photosynthetic reaction centers.

PACS numbers: 31.70.Hq, 82.30.Fi, 87.15.Rn

\section{Introduction}

Photoinduced reactions in multicenter supramolecular systems may lead to many excited products of charge transfer (CT) character. These systems are particularly important in the field of charge separation. The photochemical processes involving the $\mathrm{CT}$ states are dynamic processes occurring on multidimensional potential energy surfaces involving intramolecular coordinates and solvent polarization coordinates. After photoexcitation many dyes and photobiological systems undergo

*The results of this paper were initially presented at The Jablonski Centennial Conference on Luminescence and Photophysics, July 23-27, 1998, Torun, Poland.

$\dagger_{\text {e-mail: ucnajbar@kinga.cyf-kr.edu.pl }}$ 
photochemical transformations which lead to one of many possible excited states of CT character [1-8]. Rettig and co-workers have discussed the problems of the multidimensional photochemistry in flexible dye systems $[4,7]$. The photophysics and the photochemistry of the donor-spacer-acceptor and donor-donor-acceptor systems in polar solvents have been investigated by Warman and co-workers [9] and Verhoeven et al. [10-12]. These photochemical reactions involve both large amplitude changes in the internal coordinates as well as substantial outer sphere reorganization of the solvent. The role of the internal reorganization in the photoreactions and the solvation of molecules in their initially excited states and the photoproducts varies depending on the flexibility of the molecule undergoing the photoreaction and the solvent polarity. In the rigid molecular systems having highly polar excited states of CT character strongly interacting with the solvents of high polarity the major contributions to the reaction coordinate are due to the solvation of charges or dipoles. For rigid supramolecular systems the solvent polarization coordinate represents the major reaction coordinate for the electron transfer process [13-21].

Photosynthesis is carried out by supramolecular complexes containing as chromophoric subunits chlorophylls, porphyrins, quinones and carotenoid polyenes in a protein environment $[8,17,18,22-26]$. Photoexcitation of the photosynthetic reaction center initiates a series of ultrafast photophysical and photochemical events leading to charge separation used to drive slower chemical transformations. The antenna system consisting of chlorophyll and carotenoides transfers the electronic excitation to the chlorophyll dimer. These processes are ultrafast, occurring on the $100 \mathrm{fs}$ timescale $[22,27,28]$. In the photosynthetic reaction center, e.g., Rhodopseudomonas viridis, a bacteriochlorophyll special pair donor $(P)$ is initially excited. The electron is transferred within $3.6 \mathrm{ps}$ from $\left(P^{*}\right)$ to a distant bacterio-pheophytin $\left(H_{L}\right)[24 ; 25,29]$. These two subunits are bridged by a bacteriochlorophyll monomer $\left(B_{L}\right)$, which can function as a real electron acceptor and can contribute to the effective electronic coupling between the special pair dimer $\left(P^{*}\right)$ and bacteriopheophytin $\left(H_{L}\right)$. The electron is subsequently transferred to quinones. In the final stage a long-lived transmembrane charge separation is achieved.

The multistep electron transfer processes in the photosynthetic reaction centers are characterised by the quantum yields close to 1 . These high quantum efficiencies of the energy and electron transfer processes in the photosynthetic reaction centers are determined by the electronic energy levels of the constituting subunits, their photophysical and redox properties, separations and mutual orientations in space and the electronic and dynamical properties of the bridging medium $[22,30,31]$. There is a continuous interest in the understanding of the role of different factors influencing the charge separation rates and the resulting efficiencies of the initial steps of the charge separation. Zinth et al. $[8,32]$ have found evidence for the appearance of $B_{L}^{-}$as a distinct chemical intermediate which forms with $\tau=3.5 \mathrm{ps}$ and disappears with $\tau=0.9 \mathrm{ps}$. Recent kinetic analysis of the charge separation in the bacterial photosynthetic center by Bixon et al. seems to support this interpretation [27]. 
There are also significant advances in synthesis of the supramolecular systems that mimic some functions of the photosynthetic reaction center $[8,22,33-45]$. The goal is to achieve a long-lived and efficient charge separation between the distant subunits in an environment which differs from that in biological systems. The investigation of the factors influencing the rates of the charge recombination processes is particularly important for achieving a high quantum yield and long-lived charge separation. Osuka et al. [37-40] have developed models of the photosynthetic reaction center consisting of 2,3 , and 4 porphyrins with an individual benzoquinone or pyromellitimide acceptor attached to the terminal porphyrin. Two porphyrins are stacked to act like the special pair dimer in photosynthetic systems. They have shown that rapid electron transfer occurs between the porphyrin and the acceptors when they are adjacent one to another.

Important information concerning the potential energy functions in the triad systems has been obtained using molecular dynamics simulation for the photosynthetic reaction centers [26, 46-48]. The simulations performed by Marchi et al. [46] for the bacterial photosynthetic reaction center, Rhodopseudomonas viridis, have shown that the fluctuations in the two energy gaps relevant to the initial charge separation are strongly correlated. In recent papers [49-51] it has been demonstrated that the competitive electron transfer processes in the supramolecular triad system can be modeled using the potential functions in two solvent polarization coordinates. The basic parameters determining the shape of the potential surfaces are the reorganization energies for each coordinate and their correlation coefficient.

The purpose of this paper is as follows: (i) we analyse the time evolution of the reaction rates in the triad system, (ii) we analyse the time evolution of contribution due to superexchange mechanism of the electron transfer, (iii) we analyse experimental data for Rhodopseudomonas viridis and Rhodobacter sphaeroides, (iv) we show that the electron transfer rates can be satisfactorily reproduced assuming dephasing rates comparable to those observed for the electronic transitions in dye molecules in polar solvents.

\section{Potential energy surfaces}

The charge separation in the triad system involves three electronic states (1) $D-A-A^{*}$, (2) $D^{+}-A^{-}-A$, and (3) $D^{+}-A-A^{-}$. The fluctuations in the energy level differences (1) vs. (2) and (2) vs. (3) are characterised by reaction coordinates $q_{1}$ and $q_{2}$, respectively. The diabatic energy surface in these reaction coordinates $q_{1}, q_{2}[49,52-54]$ for the initial neutral state $U_{1}\left(q_{1}, q_{2}\right)$ is given by

$$
U_{1}\left(q_{1}, q_{2}\right)=\frac{1}{2\left(1-\rho^{2}\right)}\left(\frac{q_{1}^{2}}{2 \lambda_{12}}-\frac{2 \rho q_{1} q_{2}}{\sqrt{4 \lambda_{12} \lambda_{23}}}+\frac{q_{2}^{2}}{2 \lambda_{23}}\right),
$$

where $\lambda_{l k}$ are the Marcus reorganization energies $[55,56], \lambda_{k}$ are the reorganization energy parameters [57] and $\rho=\lambda_{2} / \sqrt{\lambda_{12} \lambda_{23}}$ is the correlation coefficient of the reaction coordinates. The coordinate correlation coefficient can be also given using the Marcus reorganization energies [55]

$$
\rho=\left(\lambda_{12}+\lambda_{23}-\lambda_{13}\right) /\left(2 \sqrt{\lambda_{12} \lambda_{23}}\right) .
$$




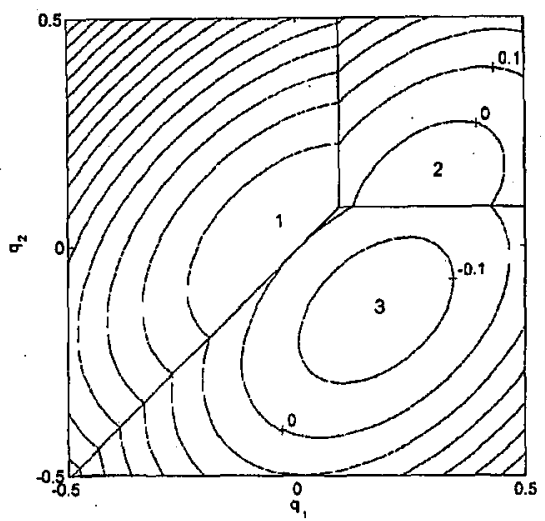

Fig. 1. Potential energy surfaces for model $I$.

Within the framework of the linear response theory the potential energy surfaces for the CT states can be obtained from $U_{1}\left(q_{1}, q_{2}\right)$. In particular, the potential energy functions for the $D^{+}-A_{1}^{-}-A_{2}$ and $D^{+}-A_{1}-A_{2}^{-}$states are given by

$$
U_{2}\left(q_{1}, q_{2}\right)=U_{1}\left(q_{1}, q_{2}\right)-q_{1}+\lambda_{12}+\Delta G_{12}
$$

and

$$
U_{3}\left(q_{1}, q_{2}\right)=U_{1}\left(q_{1}, q_{2}\right)-q_{1}+q_{2}+\lambda_{13}+\Delta G_{13}
$$

respectively. The minimum of the surface $U_{2}\left(q_{1}, q_{2}\right)$ is located at $q_{1}^{(2)}=2 \lambda_{12}=$ $2 \lambda_{1}+2 \lambda_{2}, q_{2}^{(2)}=2 \lambda_{2}$; the minimum of the surface $U_{3}\left(q_{1}, q_{2}\right)$ is at $q_{1}^{(2)}=$ $2 \lambda_{12}-2 \lambda_{2}=2 \lambda_{1}, q_{2}^{(3)}=-2 \lambda_{23}+2 \lambda_{2}=-2 \lambda_{3}$. The potential functions for the three electronic states in the triad system are shown in Fig. 1 in a contour plot. The lowest energy surface is shown in each coordinate area. The intersections of the potential energy surfaces are shown as solid straight lines. The potential energies shown in Fig. 1 have been derived from the results obtained by Marchi et al. [46] for the photosynthetic reaction center Rhodopseudomonas viridis using molecular dynamics calculations. The parameters used in the modeling are given in Table.

\section{Stochastic Liouville equation for the three surface systems}

The electronic interactions between the different electronic states determine the electron transfer rates. In general, many electronic states can contribute to the electron transfer between the initial and final electronic states. Electronic states localised predominantly on the bridging subunits can function as virtual electronic states contributing to the superexchange mechanism. The importance of the different electronic states depends on the separation of the energy levels involved in electron transfer. In polar solvents the fluctuations in the energy level separations make the electron transfer depend on the state of instantaneous solvent polarization. These variations in the electronic state separations for the supramolecular system in a polar environment can be accounted using the potential energy functions with the appropriate number of the solvent polarization coordinates. The 
TABLE

Reorganization energies and electronic coupling parameters.

\begin{tabular}{l|c|c|c}
\hline Parameter & Model I & Model II & Model III \\
\hline$\lambda_{12}$ & $0.1432 \mathrm{eV}$ & $0.0991 \mathrm{eV}$ & $0.0800 \mathrm{eV}$ \\
$\lambda_{23}$ & $0.1273 \mathrm{eV}$ & $0.0881 \mathrm{eV}$ & $0.0771 \mathrm{eV}$ \\
$\Delta G_{13}$ & $-0.15 \mathrm{eV}$ & $-0.17 \mathrm{eV}$ & $-0.17 \mathrm{eV}$ \\
$\Delta G_{12}$ & $-0.25 \ldots 0.25 \mathrm{eV}$ & $-0.2 \ldots 0.1 \mathrm{eV}$ & $-0.2 \ldots 0.1 \mathrm{eV}$ \\
$\rho$ & 0.421 & 0.421 & 0.421 \\
$V_{13}$ & 0.0 & 0.0 & 0.0 \\
$V_{23}$ & $0.012 \mathrm{eV}$ & $0.012 \mathrm{eV}$ & $0.012 \mathrm{eV}$ \\
$V_{12}$ & $0.002 \mathrm{eV}$ & fitted & $0.0026 \mathrm{eV}$
\end{tabular}

Marcus theory of the outer-sphere electron transfer in the donor-acceptor system considers two electronic energy levels dependent on the single solvent polarization coordinate.

The three-surface model allows already for the modeling of the sequential and superexchange electron transfer processes in the triad system. It is convenient to describe the dynamics of the three level system using the density matrix method. The stochastic Liouville equation for the density matrix for the three level system has the following form:

$$
\frac{\partial}{\partial t} \rho\left(q_{1}, q_{2}, t\right)=-\frac{\dot{i}}{\hbar}\left[H\left(q_{1}, q_{2}, t\right), \rho\left(q_{1}, q_{2}, t\right)\right]+\left.\frac{\partial}{\partial t} \rho\left(q_{1}, q_{2}, t\right)\right|_{\text {rel }},
$$

where the system Hamiltonian is given by

$$
H\left(q_{1}, q_{2}, t\right)=\left[\begin{array}{ccc}
U_{1}\left(q_{1}, q_{2}\right) & V_{12} & V_{13} \\
V_{12} & U_{2}\left(q_{1}, q_{2}\right) & V_{23} \\
V_{13} & V_{23} & U_{3}\left(q_{1}, q_{2}\right)
\end{array}\right] .
$$

Here $V_{m n}$ are the electronic coupling matrix elements between the diabatic potential energy surfaces $U_{m}\left(q_{1}, q_{2}\right)$ and $U_{n}\left(q_{1}, q_{2}\right)$, respectively. $U_{m n}=$ $U_{m}\left(q_{1}, q_{2}\right)-U_{n}\left(q_{1}, q_{2}\right)$ are the vertical energy differences. The following relation holds: $U_{12}+U_{23}=U_{13}$.

The relaxation term takes into account solvation dynamics on the three surfaces and phenomenologically includes the dephasing for the off diagonal elements of the density matrix

$$
\left.\frac{\partial}{\partial t} \rho\left(q_{1}, q_{2}, t\right)\right|_{\mathrm{rel}}=\left[\begin{array}{ccc}
\Gamma_{11}^{D} \circ \rho_{11} & -\Gamma_{12} \rho_{12} & -\Gamma_{13} \rho_{13} \\
-\Gamma_{21} \rho_{21} & \Gamma_{22}^{D} \circ \rho_{22} & -\Gamma_{23} \rho_{23} \\
-\Gamma_{31} \rho_{31} & -\Gamma_{32} \rho_{32} & \Gamma_{\mathbf{3 3}}^{D} \circ \rho_{33}
\end{array}\right]:
$$

The form of the Hamiltonian and the relaxation term imply that in the present formulation we can model both the static limit of the solvent fluctuations and homogeneous pure dephasing for the decay of electronic coherence. 
The matrix elements of the density in the frequency domain satisfy the following equations:

$$
\overline{s \rho}_{m, n}-\rho_{m n}(0)=-\frac{\mathrm{i}}{\hbar}[h, \bar{\rho}]_{m n}+L\left\{\partial \rho_{m n} /\left.\partial t\right|_{\mathrm{rel}}\right\}, \quad m, n=1,2,3 .
$$

Using the solutions for the off-diagonal matrix elements the kinetic equations for the populations of the three levels in the Laplace domain can be written in terms of the three rate functions

$$
\begin{gathered}
s \bar{\rho}_{11}\left(q_{1}, q_{2}, s\right)-\rho_{11}(0)=-F_{1}\left(q_{1}, q_{2}, s\right)-\bar{F}_{2}\left(q_{1}, q_{2}, s\right)+\Gamma_{11}^{D} \bar{\rho}_{11}\left(q_{1}, q_{2}, s\right) \\
s \bar{\rho}_{22}\left(q_{1}, q_{2}, s\right)=\bar{F}_{1}\left(q_{1}, q_{2}, s\right)-\bar{F}_{3}\left(q_{1}, q_{2}, s\right)+\Gamma_{22}^{D} \bar{\rho}_{22}\left(q_{1}, q_{2}, s\right), \\
s \bar{\rho}_{33}\left(q_{1}, q_{2}, s\right)=\bar{F}_{2}\left(q_{1}, q_{2}, s\right)+\bar{F}_{3}\left(q_{1}, q_{2}, s\right)+\Gamma_{33}^{D} \bar{\rho}_{33}\left(q_{1}, q_{2}, s\right) .
\end{gathered}
$$

The rate functions have been defined as follows:

$$
\begin{aligned}
& \bar{F}_{1}\left(q_{1}, q_{2}, s\right)=\bar{K}_{1}\left(q_{1}, q_{2}, s\right)\left[\bar{\rho}_{11}\left(q_{1}, q_{2}, s\right)-\bar{\rho}_{22}\left(q_{1}, q_{2}, s\right)\right], \\
& \bar{F}_{2}\left(q_{1}, q_{2}, s\right)=\bar{K}_{2}\left(q_{1}, q_{2}, s\right)\left[\bar{\rho}_{11}\left(q_{1}, q_{2}, s\right)-\bar{\rho}_{33}\left(q_{1}, q_{2}, s\right)\right], \\
& \bar{F}_{3}\left(q_{1}, q_{2}, s\right)=\bar{K}_{3}\left(q_{1}, q_{2}, s\right)\left[\bar{\rho}_{22}\left(q_{1}, q_{2}, s\right)-\bar{\rho}_{33}\left(q_{1}, q_{2}, s\right)\right],
\end{aligned}
$$

where the functions $\bar{K}_{l}\left(q_{1}, q_{2}, s\right)$ play the role of the effective coupling between the donor and acceptor sites.

The solutions of the kinetic equations for the populations of the levels in the Laplace domain in terms of the Gaussian wave packets are given by

$$
\begin{gathered}
\bar{\rho}_{11}\left(q_{1}, q_{2}, s\right)=\iint \rho_{11}\left(q_{1}^{0}, q_{2}^{0}, 0\right) \bar{\phi}_{1}\left(q_{1}, q_{2}, s \mid q_{1}^{0}, q_{2}^{0}\right) \mathrm{d} q_{1}^{0} \mathrm{~d} q_{2}^{0} \\
-\iint \bar{F}_{1}\left(q_{1}^{0}, q_{2}^{0}, s\right) \bar{\phi}_{1}\left(q_{1}, q_{2}, s \mid q_{1}^{0}, q_{2}^{0}\right) \mathrm{d} q_{1}^{0} \mathrm{~d} q_{2}^{0} \\
-\iint \bar{F}_{2}\left(q_{1}^{0}, q_{2}^{0}, s\right) \bar{\phi}_{1}\left(q_{1}, q_{2}, s \mid q_{1}^{0}, q_{2}^{0}\right) \mathrm{d} q_{1}^{0} \mathrm{~d} q_{2}^{0}, \\
\bar{\rho}_{22}\left(q_{1}, q_{2}, s\right)=\iint \bar{F}_{1}\left(q_{1}^{0}, q_{2}^{0}, s\right) \bar{\phi}_{2}\left(q_{1}, q_{2}, s \mid q_{1}^{0}, q_{2}^{0}\right) \mathrm{d} q_{1}^{0} \mathrm{~d} q_{2}^{0} \\
-\iint \bar{F}_{3}\left(q_{1}^{0}, q_{2}^{0}, s\right) \bar{\phi}_{2}\left(q_{1}, q_{2}, s \mid q_{1}^{0}, q_{2}^{0}\right) \mathrm{d} q_{1}^{0} \mathrm{~d} q_{2}^{0}, \\
\bar{\rho}_{33}\left(q_{1}, q_{2}, s\right)=\iint \bar{F}_{2}\left(q_{1}^{0}, q_{2}^{0}, s\right) \bar{\phi}_{3}\left(q_{1}, q_{2}, s \mid q_{1}^{0}, q_{2}^{0}\right) \mathrm{d} q_{1}^{0} \mathrm{~d} q_{2}^{0} \\
+\iint \bar{F}_{3}\left(q_{1}^{0}, q_{2}^{0}, s\right) \bar{\phi}_{3}\left(q_{1}, q_{2}, s \mid q_{1}^{0}, q_{2}^{0}\right) \mathrm{d} q_{1}^{0} \mathrm{~d} q_{2}^{0},
\end{gathered}
$$

where the $\bar{\phi}_{n}\left(q_{1}, q_{2}, s \mid q_{1}^{0}, q_{2}^{0}\right)$ are the Laplace transforms of the functions $\phi_{n}\left(q_{1}, q_{2}, t \mid q_{1}^{0}, q_{2}^{0}\right)$ describing the dynamics of the system in $n$-th electronic state in the absence of electron transfer. The Laplace transforms $\bar{\phi}_{n}\left(q_{1}, q_{2}, s \mid q_{1}^{0}, q_{2}^{0}\right)$ contain 
contributions arising from the equilibrium distribution of the reaction coordinates and the relaxation term

$$
\bar{\phi}_{n}\left(q_{1}, q_{2}, s \mid q_{1}^{0}, q_{2}^{0}\right)=\phi_{n}^{\mathrm{eq}}\left(q_{1}, q_{2}\right) / s+\phi_{n}^{\mathrm{eq}}(0,0) \bar{\tau}_{n}\left(q_{1}, q_{2}, s \mid q_{1}^{0}, q_{2}^{0}\right) .
$$

Relaxations in the reaction coordinate space are represented here by the system timescale functions $\bar{\tau}_{n}\left(q_{1}, q_{2}, s \mid q_{1}^{0}, q_{2}^{0}\right)$.

From Eqs. (3.5)-(3.7) the integral equations for the rate functions $\bar{F}_{l}\left(q_{1}, q_{2}, s\right)$ can be obtained. The solutions of these equations allow for a detailed description of the dynamics of electron transfer processes in the three-surface system. In the most common situations total populations of the electronic states are sufficient. The total populations of the three levels in the Laplace domain can be obtained by integrating the populations $\bar{\rho}_{m n}\left(q_{1}, q_{2}\right)$ over the solvent polarization coordinates

$$
\bar{\rho}_{m n}(s)=\iint \bar{\rho}_{m n}\left(q_{1}, q_{2}\right) \mathrm{d} q_{1} \mathrm{~d} q_{2} .
$$

The functions

$$
\bar{F}_{m}(s)=\iint \bar{F}_{m}\left(q_{1}, q_{2}, s\right) \mathrm{d} q_{1} \mathrm{~d} q_{2}
$$

represent the total rate functions for the transitions between three electronic levels in the triad system.

The nonadiabatic limit of the electron transfer processes in the triad system holds when all the system timescale functions vanish, $\bar{\tau}_{n}\left(q_{1}, q_{2}, s \mid q_{1}^{0}, q_{2}^{0}\right) \approx 0$ [52]. In this limiting case all integral terms containing the functions $\bar{\tau}_{n}\left(q_{1}, q_{2}, s \mid q_{1}^{0}, q_{2}^{0}\right)$ vanish and we obtain a simple set of algebraic equations for the Laplace transforms of the rate functions $\bar{F}_{n}(s)$ :

$$
[\overline{\boldsymbol{K}}(s)+s \boldsymbol{I}] \times \overline{\boldsymbol{F}}(s)=\overline{\boldsymbol{I}}_{\mathrm{ini}}(s),
$$

where $\overline{\boldsymbol{F}}(s)^{\mathrm{T}}=\left\{\bar{F}_{1}(s), \bar{F}_{2}(s), \bar{F}_{3}(s)\right\}$ and $\boldsymbol{I}$ is the unit matrix. The vector $\overline{\boldsymbol{I}}_{\text {ini }}(s)^{\mathrm{T}}=$ $\left.\left\{\bar{Q}_{11}(s), \bar{Q}_{21}(s), 0\right)\right\}$ is determined by the initial distribution of the reaction coordinates, where

$$
\bar{Q}_{m n}(s)=\iint \bar{K}_{m}\left(q_{1}, q_{2}, s\right) \phi_{n}^{\mathrm{eq}}\left(q_{1}, q_{2}\right) \mathrm{d} q_{1} \mathrm{~d} q_{2} .
$$

The kinetic matrix $\overline{\boldsymbol{K}}(s)$ is given by

$$
\overrightarrow{\boldsymbol{K}}(s)=\left[\begin{array}{ccc}
\bar{Q}_{11}(s)+\bar{Q}_{12}(s) & Q_{11}(s) & -Q_{12}(s) \\
\bar{Q}_{21}(s) & \bar{Q}_{21}(s)+\bar{Q}_{23}(s) & \bar{Q}_{23}(s) \\
-Q_{32}(s) & \bar{Q}_{33}(s) & \bar{Q}_{32}(s)+\bar{Q}_{33}(s)
\end{array}\right]
$$

The solution of the coupled equations for the rate functions can be obtained using the inverse matrix $[\overline{\boldsymbol{K}}(s)+s \boldsymbol{I}]^{-1}$ and numerical inverse Laplace transformation. 


\section{The coupling functions}

The functions $\bar{K}_{m}\left(q_{1}, q_{2}, s\right)$ are the solutions of the equations for off-diagonal elements of the density matrix. The functions $K_{i j}$ characterising the coupling between electronic states have significant values along the intersection of the potential surfaces when $U_{i j}$ is close to 0 . A special situation occurs near the point in coordinate space where three electronic states have the same energy. In this area we observe interference effects in the coupling functions. Outside this area the coupling functions are given by Lorentz type functions of the reaction coordinates. For the photosynthetic reaction center the distance between the donor $D$ and the second acceptor $A_{2}$ is large. The corresponding electronic coupling matrix element is expected to be very small $V_{13} \approx 0[18,24,25,58]$. We can distinguish two situations:

(i) When $V_{l k}=0$ the dominant contribution to the effective coupling between the states $l$ and $k$ is due to a coupling of the initial and final state through the intermediate state $s \neq l, s \neq k$. In the case of the superexchange mechanism the function $\bar{K}_{X}\left(q_{1}, q_{2}, s\right)$ shows a strong dependence on both reaction coordinates. The function reaches the maximum at the point common to the three intersection lines. Moreover, along the direction of the two other intersection lines it attains the negative values. The contour plot of $\bar{K}_{2}\left(q_{1}, q_{2}, s\right)$ is shown in Fig. 2 . The properties of the coupling function $\bar{K}_{2}\left(q_{1}, q_{2}, s\right)$ for the model I are shown in Fig. $2 \mathrm{~b}$ and $\mathrm{d}$ for two values of the dephasing parameter $\Gamma=3$ and $10\left[\mathrm{ps}^{-1}\right]\left(\Gamma=\Gamma_{12}=\Gamma_{23}=\Gamma_{13}\right)$. Other parameters belong to set $\mathrm{I}$ in Table. The coupling function shows two peaks
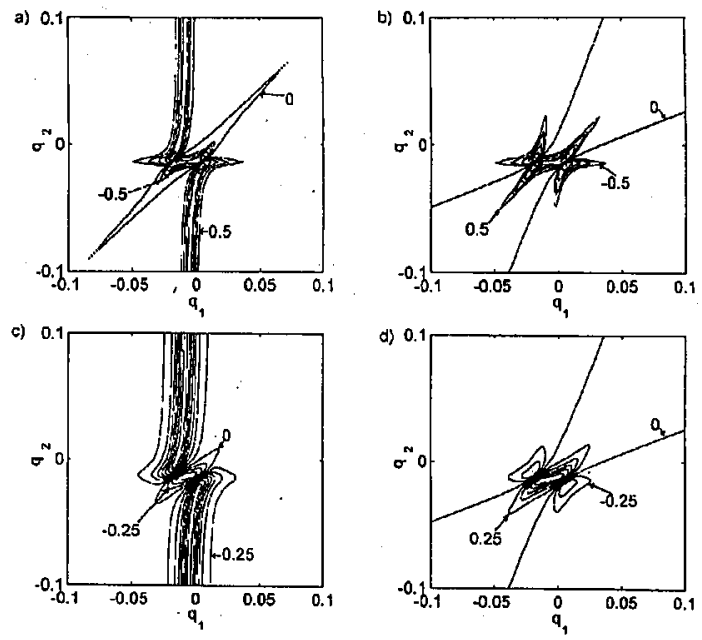

Fig. 2. The coupling functions for model I, when $V_{12}=0.002 \mathrm{eV}$; (a) $K_{1}\left(q_{1}, q_{2}\right)$ for $\Gamma=3 \mathrm{ps}^{-1}$, (b) $K_{2}\left(q_{1}, q_{2}\right)$ for $\Gamma=3 \mathrm{ps}^{-1}$, (c) $K_{1}\left(q_{1}, q_{2}\right)$ for $\Gamma=10 \mathrm{ps}^{-1}$, (d) $K_{2}\left(q_{1}, q_{2}\right)$ for $\Gamma=10 \mathrm{ps}^{-1}$. 
and two minima which are located off the intersection lines of the potential energy surfaces. With increasing dephasing rate the effective coupling function

$$
\bar{K}_{2}\left(q_{1}, q_{2}, s\right)=\frac{V_{12} V_{23}}{\hbar^{2}} \bar{S}_{2}\left(q_{1}, q_{2}, s\right)
$$

decreases and spreads over a large area in coordinate space.

(ii) When $V_{l k} \neq 0$ the dominant contribution is due to a direct coupling between the states $1 \Rightarrow 2$ and $2 \Rightarrow 3$. The corresponding rate functions $\bar{K}_{X}\left(q_{1}, q_{2}, s\right)$, $X=1,2$, can be given in the following form:

$$
\bar{K}_{X}\left(q_{1}, q_{2}, s\right)=\frac{V_{l k}^{2}}{\hbar^{2}} \bar{P}_{X}\left(q_{1}, q_{2}, s\right)-\frac{V_{12} V_{23}}{\hbar^{2}} \bar{S}_{2}\left(q_{1}, q_{2}, s\right) .
$$

Far from the point of the degeneracy of the three surfaces we can use the Zusman approximation [59] to obtain

$$
\bar{K}_{X}\left(q_{1}, q_{2}, s\right) \approx \frac{V_{l k}^{2}}{\hbar^{2}} \bar{P}_{X}\left(q_{1}, q_{2}, s\right) \approx \frac{2 \pi V_{l k}^{2}}{\hbar} \delta\left(U_{l k}\right) .
$$

The properties of the coupling function $\bar{K}_{1}\left(q_{1}, q_{2}, s\right)$ for the model I are shown in Fig. 2a and $\mathrm{c}$ for two values of the dephasing parameter $\Gamma=3$ and $10\left[\mathrm{ps}^{-1}\right]$. This function is negative in the area of near degeneracy of the three surfaces. In this area the dominant positive contribution involves the coupling function $\bar{K}_{2}\left(q_{1}, q_{2}, s\right)$ determining the rate of the superexchange process.

\section{Time dependent rates}

The rate functions $F_{j}(t), j=1,2$, and 3 , calculated for the set I of the parameters are shown in Fig. 3. The free energy difference $\Delta G_{13}=-0.15 \mathrm{eV}$ gives a barrierless situation for the direct electron transfer process (1)-(3). In Fig. 3 the $F_{j}(t)$ are plotted as a function of time and of the free energy for the electron transfer between electronic states (1) and (2), $\Delta G_{12}$. The observed time dependences of the electron transfer rates $F_{1}(t)$ and $F_{2}(t)$ represent nearly exponential decays. The greatest rates occur for the barrierless situation between states (1) and (2). For longer times significant electron transfer rates $F_{1}(t)$ can be found for $\Delta G_{i j} \approx 0$. For longer times and for a free energy condition favoring the sequential process the rate functions $F_{1}(t)$ and $F_{3}(t)$, shown in Fig. 3a and $c$, are very close to each other as should occur for the consecutive reactions. The rate function $F_{2}(t)$, Fig. $3 \mathrm{~b}$, representing the electron transfer rate due to superexchange is small under these conditions. However, relative contributions of the sequential process and the superexchange process depend both on free energy and on time. The calculations show that electronic states dephasing processes have small influence on the superexchange contribution to the charge separation process, $F_{2}(t)$. The increase in the dephasing rate increases the contribution due to the sequential mechanism, $F_{1}(t)$.

The relative contribution of the superexchange mechanism in the charge separation process can be quantitatively characterised using these two functions $F_{1}(t)$ and $F_{2}(t)$. For comparison two different functions can be defined: 


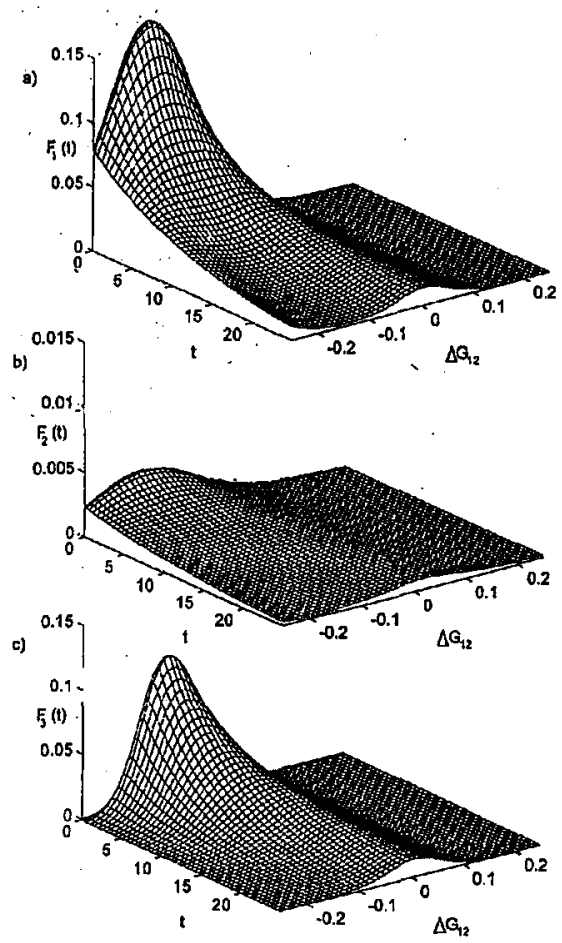

Fig. 3. Time dependent rate functions (a) $F_{1}(t)$, (b) $F_{2}(t),\left(\right.$ c) $F_{3}(t)$ for different values of $\Delta G_{12}$. Set of parameters for model I, $\Gamma=3 \mathrm{ps}^{-1}$.

$$
R(t)=\frac{F_{2}(t)}{F_{1}(t)+F_{2}(t)}
$$

and

$$
R_{I}(t)=\int_{0}^{t} F_{2}(t) \mathrm{d} t / \int_{0}^{t}\left[F_{1}(t)+F_{2}(t)\right] \mathrm{d} t .
$$

The first function $R(t)$ shows the relative contribution of the electron transfer rate due to the superexchange process to the total rate of the decay of the initial state. The second function $R_{I}(t)$ defines the corresponding contribution in terms of the fraction of the initial state population decay occurring due to the superexchange. The dependences of these two characteristics $R(t)$ and $R_{I}(t)$ on time and on the free energy of the intermediate state are shown in Fig. $4 \mathrm{a}$ and b, respectively. The ratio $R(t)$ shows some time variations under barrierless situations. For long times special features occur around the free energy range $\Delta G_{12} \approx-\lambda_{12}$ and for short time at $\Delta G_{12} \approx \lambda_{12}$. In this latter case the back electron transfer $(2) \rightarrow(1)$ is a barrierless process. Using the integral function $R_{I}(t)$ only a strong free energy dependence of the superexchange contribution is seen, Fig. $4 \mathrm{~b}$. The limiting value of the superexchange ratio at high positive values of the free energy $\Delta G_{12}$ shows a clear dependence on the dephasing rate. This dependence of the superexchange process on the dephasing parameter is shown in Fig. 4c. The limiting 

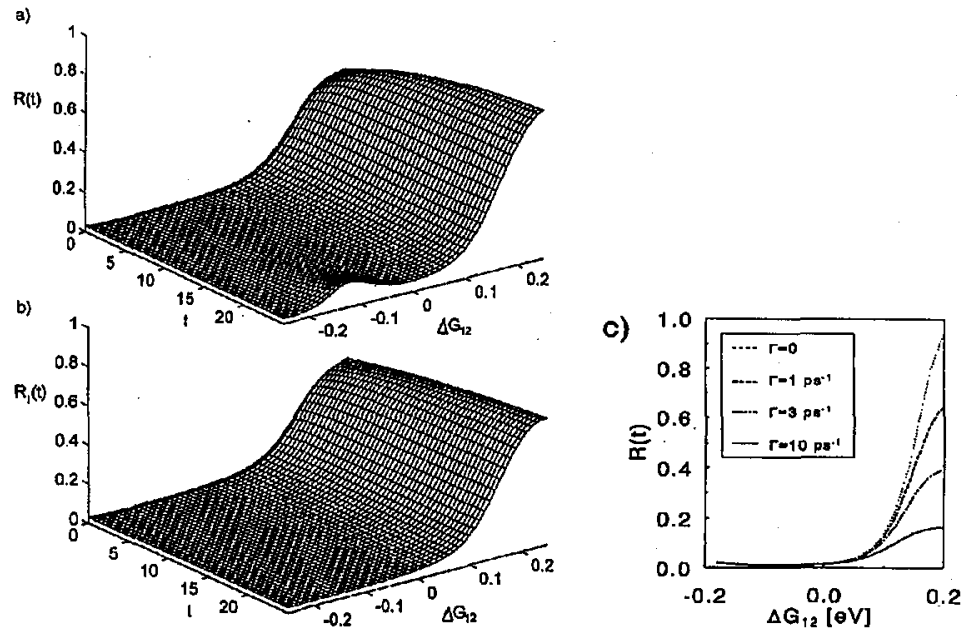

Fig. 4. Superexchange ratio calculated from (a) Eq. (5.1) $-R(t)$ and (b) Eq. (5.2) $-R_{I}(t)$, for model I, $\Gamma=3 \mathrm{ps}^{-1}$; (c) superexchange ratio $R_{I}\left(\Delta G_{12}\right)$ calculated for different values of $\Gamma$ and time $t=15 \mathrm{ps}$.

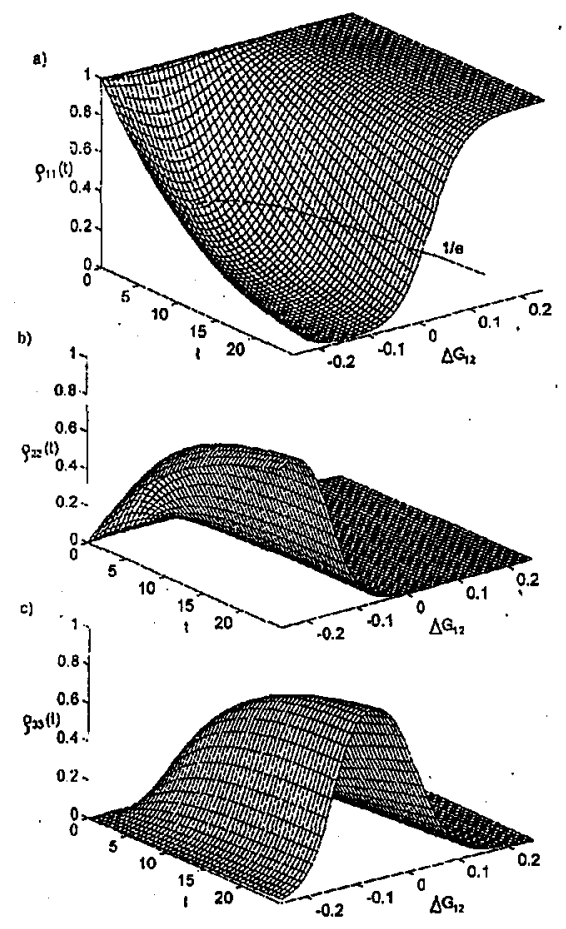

Fig. 5. Calculated populations of states (a) 1, (b) 2 and (c) 3; model I, $\Gamma=3 \mathrm{ps}^{-1}$. Plotted line $\rho_{11}\left(t, \Delta G_{12}\right)=1 / e$ in part (a) allows to determine state 1 decay time. 
value of the superexchange ratio $R(t)=1$ can be obtained only for a negligible dephasing rate. The dephasing processes reduce the relative contribution of the superexchange mechanism of the charge separation in the triad system.

The electron transfer rate functions $F_{j}(t)$ allow for calculation the time evolution of the populations of the three electronic states. These are given by the following formulae:

$$
\begin{aligned}
& \rho_{11}(t)=1-\int_{0}^{t} F_{1}(\tau) \mathrm{d} \tau-\int_{0}^{t} F_{2}(\tau) \mathrm{d} \tau \\
& \rho_{22}(t)=\int_{0}^{t} F_{1}(\tau) \mathrm{d} \tau-\int_{0}^{t} F_{3}(\tau) \mathrm{d} \tau \\
& \rho_{33}(t)=\int_{0}^{t} F_{2}(\tau) \mathrm{d} \tau+\int_{0}^{t} F_{3}(\tau) \mathrm{d} \tau .
\end{aligned}
$$

The time dependences of the populations of the excited states are given in Fig. $5 \mathrm{a}-\mathrm{c}$ for different values of the free energy $\Delta G_{12}$. We find that for the parameters of set I in Table the charge separation rates show a weak dependence on $\Delta G_{13}$.

\section{The decay rate constants}

The calculated time dependent population of the initial state allows us to determine several other features of the system. From the slopes of $\ln \left[F_{1}(t)+F_{2}(t)\right]$ as a function of time, rate constants for the decay of the initial state can be derived. Extremely fast charge separation occurs under barrierless conditions. The calculated decay rate constants for the initial state are shown in Fig. 6a. The dependence of these rate constants on the energy of the intermediate state $\Delta G_{12}$ in the vicinity of the maximum can be approximated by a Gaussian line shape. The change in rate with the dephasing rate in this Gaussian region can be described by changes in the width of the Gaussian. Thus the influence of the dephasing can be accounted for by changes in the reorganization energy. These changes are relatively small, reaching less than $15 \%$ for physically reasonable changes of the
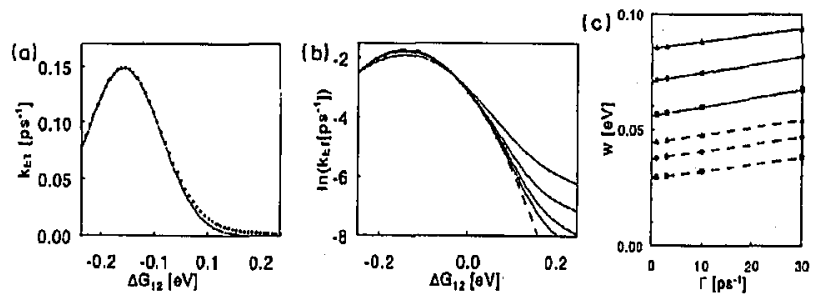

Fig. 6. (a) Dependence of state 1 decay rate constant on free energy $\Delta G_{12}$. (b) The same dependence in logarithmic scale. (c) Influence of dephasing constant $\Gamma$ on the width of the Gaussian component: $V_{12}=0.0025 \mathrm{eV}$, full $\Delta \cdot \lambda_{12}=0.1432 \mathrm{eV}, \bullet \lambda_{12}=0.0991 \mathrm{eV}$, - $\lambda_{12}=0.0662 \mathrm{eV}$; solid lines: $T=293 \mathrm{~K}$, dashed lines: $T=80 \mathrm{~K}$. 
dephasing rates. The width of the Gaussian region shows a linear dependence on the dephasing rate constant $\Gamma$. This is shown in Fig. $6 \mathrm{c}$ for three values of the reorganization energy, at $80 \mathrm{~K}$ and at $293 \mathrm{~K}$. For a dephasing rate $\Gamma=0$ the width of the Gaussian component reduces to the value predicted by the Marcus theory of the nonadiabatic electron transfer [55]. For large dephasing rates and positive values of $\Delta G_{12}$ the calculated electron transfer rates are higher than those predicted from the extrapolation of the Gaussian component of the line shape. The wings of the line shape function cannot be accounted for by the Gaussian function. This is shown in Fig. 6b for four dephasing rates. Under these conditions the electronic dephasing acts to promote sequential charge separation. This conclusion agrees with the calculations presented in Fig. $4 \mathrm{a}$ and b. They show that the electronic dephasing processes lead to the decrease in the relative contribution of the superexchange mechanism in the charge separation process.

The broadening of the dependence of the electron transfer rate constant on the energy of the intermediate state $\Delta G_{12}$ with the rates of dephasing is a direct consequence of the broadening of the coupling functions $\bar{K}_{X}\left(q_{1}, q_{2}, s\right)$. Electron transfer processes are not limited to the intersection regions of the potential energy surfaces but occur over a much larger area of the reaction coordinate space. The broadening of the coupling functions have a smaller effect on the electron transfer occurring with a small energy barrier (represented by central Gaussian component) than on that requiring a high activation energy. In this latter case the overlap of the broadened coupling functions $\bar{K}_{X}\left(q_{1}, q_{2}, s\right)$ with the Gaussian equilibrium distribution functions of the reaction coordinates $\bar{Q}_{m n}(s)$ decrease with an increase in $\Delta G_{12}$ more slowly than it is predicted by the standard Marcus theory. Under these conditions the wings of the line shape function have a Lorentzian character.

In the present calculations for the electron transfer rates in the nonadiabatic limit, the electronic dephasing in the three-level system is characterized using time independent dephasing rate constants $(\Gamma)$. However, for a two-level system it has been shown that the inclusion of the reaction coordinate relaxation results in the line narrowing effect $[60,61]$. The central part of the line shape function is narrowed. A similar effect of line narrowing should also occur in the three-level system for a finite relaxation rate of the reaction coordinates.

\section{Charge separation in the bacterial photosynthetic reaction center}

In the late 80 's the charge separation in photosynthetic centers was interpreted according to superexchange mechanism. During last few years the role of the sequential process has often been considered. Nagarajan et al. [25] have investigated the photoinduced electron transfer in the photosynthetic reaction center of Rhodobacter sphaeroides in mutant strains in which tyrosine (M)210 is replaced by phenylalanine, isoleucine, or tryptophan, at $293 \mathrm{~K}$ and $80 \mathrm{~K}$. Nagarajan et al. [25] analyse their experiments assuming sequential electron transfer using potential energy surfaces in two strongly correlated reaction coordinates. They conclude that the temperature dependence of the electron transfer reaction in the mutants cannot be explained adequately on the assumption that the mutations only alter the overall $\Delta G_{13}$ but it can be accounted for by assuming that they also increase the free energy of the additional state $\left(P^{+} B_{L}^{-}\right)$that serves as both a kinetic and a virtual 
intermediate. The evaluated increases in the free energy of $\left(P^{+} B_{L}^{-}\right)$were found to be greater than the measured changes in the free energy of $\left(P^{+} H_{L}^{-}\right)$. Recently, these topics were carefully investigated for the mutants of the Rhodopseudomonas viridis by Zinth et al. [62].

In this section we show that in the nonadiabatic limit of electron parameters allowing modeling of the experimental energy dependences can be readily chosen. Molecular dynamics simulations of the fluctuations in the electronic energy level separations for the photosynthetic reaction center give the potential energy functions and the time correlation functions of the reaction coordinates. The results derived from the molecular dynamics calculations are not absolute. Marchi et al. proposed scaling of the energy parameters to reproduce the observed in experiment energy difference between the initial state and the final state for Rhodopseudomonas viridis. Since the correlation coefficient of the reaction coordinates can be expressed as a ratio of the reorganization energy parameters it is independent of the energy scaling procedure $[46,63]$.

Other authors have used an alternative approach for estimation of the reorganization energies. The proposed values of the reorganization energies for the bacterial photosynthetic reaction centers vary over one order of magnitude from 200 to $2000 \mathrm{~cm}^{-1}$ [24-26, 46, 64]. Bixon et al. [27] estimated the reorganization energy for the first step of the charge separation comparing the charge separation rates for a large number of mutants of Rhodobacter sphaeroides. They propose a value for the reorganization energy of $800 \pm 250 \mathrm{~cm}^{-1}(0.10 \pm 0.03 \mathrm{eV})$. This value is smaller than that derived from molecular dynamics simulations for the Rhodopseudomonas viridis, i.e. $0.1432 \mathrm{eV}$ (see the model I in Table). In Table other sets of parameters are also given. The parameters of the model II were generated using a value $0.10 \mathrm{eV}$ for the reorganization energy of the first charge separation step and scaling accordingly to other reorganization energies from the model I. This scaling of the reorganization energies preserves the correlation, coefficient of the reaction coordinates. The results of calculations for the parameters of the model II are compared with the rates of the charge separations in the photosynthetic center of the mutants of Rhodobacter sphaeroides $[25,65,66]$ and Rhodopseudomonas viridis [62] in Fig. 7. The electronic coupling constants used for Rhodobacter sphaeroides and Rhodopseudomonas viridis are $0.0025 \mathrm{eV}$ and $0.005 \mathrm{eV}$, respectively. The dephasing rate constant was assumed to be $10 \mathrm{ps}^{-1}$ and $5 \mathrm{ps}^{-1}$ for the two species. The free energies $\Delta G_{12}$ of the electron transfer (for Rhodobacter sphaeroides) were evaluated from $P^{+} / P$ oxidation potentials using the following relation: $\Delta G_{12}=-1.72+3.22 E_{R}\left(P^{+} / P\right)$.

The free energy $\Delta G_{12}$ dependence and the temperature dependence of the charge separation rate constants for the four mutants investigated by Nagarajan et al. [25] are analysed in Fig. 8a and b. A decrease in temperature results in an increase in the charge separation rates for reactions small barriers. However, for charge separation processes requiring a larger thermal activation the decrease in the charge separation rates is observed. This behavior is readily reproduced by the present modeling using the parameters for the models II and III.

In the model III the modified reorganization energies and electronic coupling parameters are assumed to model the temperature variations of the charge separa- 


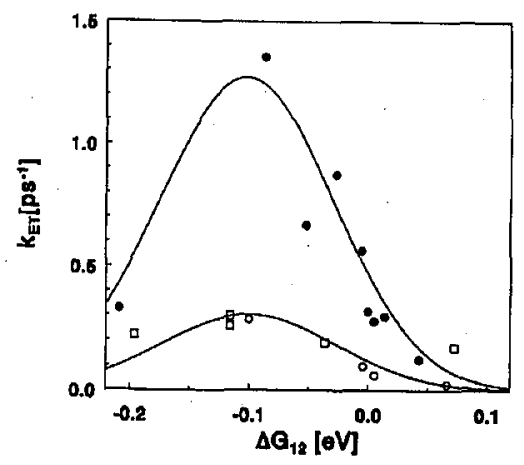

Fig. 7. The result of modeling for the bacterial photosynthetic center: - results for Rhodopseudomonas viridis, [59]; calculated curve: model II, $V_{12}=0.005 \mathrm{eV}$, $\Gamma=5 \mathrm{ps}^{-1} ; \square$, ० results for Rhodobacter sphaeroides, [25, 61]; calculated curve: model II, $V_{12}=0.0025 \mathrm{eV}, \Gamma=10 \mathrm{ps}^{-1}$; for Rhodobacter sphaeroides a relation $\Delta G_{12}=-1.72+3.22 E_{R}\left(P / P^{+}\right)$is used.
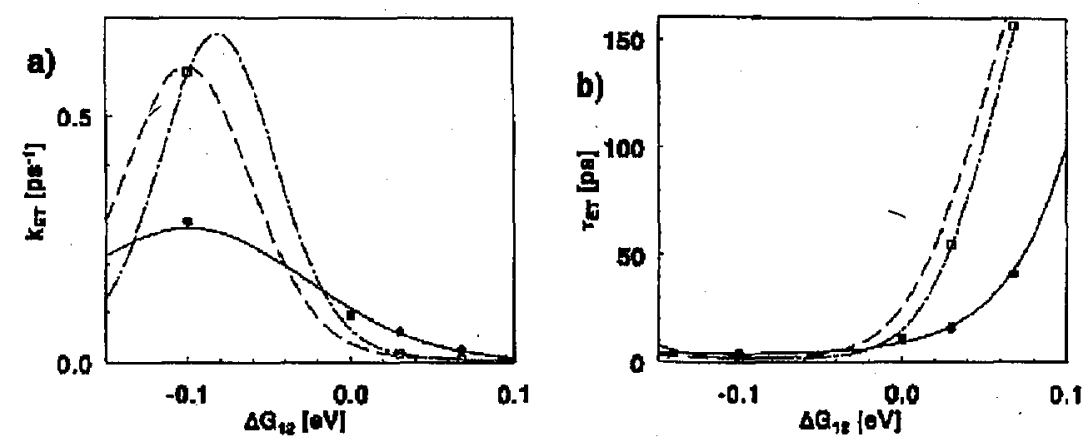

Fig. 8. The charge separation rates for Rhodobacter sphaeroides in $293 \mathrm{~K}$ and $80 \mathrm{~K}$ [25] and fitted curves for $\Gamma=10 \mathrm{ps}^{-1}$ : (a) state 1 decay rate constants, (b) state 1 decay time constants; solid line: $T=293 \mathrm{~K}$, model II, $V_{12}=0.0023 \mathrm{eV}$; dashed line: $T=80 \mathrm{~K}$, model II, $V_{12}=0.0026 \mathrm{eV}$; dash-dot line: $T=80 \mathrm{~K}, \lambda_{12}=0.080 \mathrm{eV}, V_{12}=0.0026 \mathrm{eV}$.

tion rates. The fit of the curves for two different temperatures ( 80 and $293 \mathrm{~K}$ ) we obtained by using a larger value of the electronic coupling at lower temperature. This could be explained by thermal contraction of the system. Lowering the temperature can also decrease the reorganization energies. That shifts the maximum of the curve to less negative $\Delta G_{12}$ values and also changes the amplitude and half-width.

\section{Summary}

The theory of the nonadiabatic electron transfer reactions based on the stochastic Liouville equation has been extended to triad systems, e.g., $D-A-A$. Distinctive features of the present formulation are the use of potential energy 
functions for the three electronic states using two mutually correlated solvent polarization coordinates. They describe solvent influence on the energy levels of the electronic states participating in the electron transfer processes. The characteristics of the dynamical processes are determined by potential barriers as well as the friction at the molecular level.

It seems that much more information on the role of dephasing could be obtained from the research on temperature relations of charge separation for different mutants, involving temperature dependence of reorganization energies, electronic coupling parameters, and free energies of electron transfer reactions:

Lowering temperature accelerates charge separation for free energy gaps close to reorganization energy, whereas for smaller or larger energy gaps the process is slowed by lowering the temperature. This result is consistent with experimental data.

Molecular dynamics calculations for lower temperatures may provide input on changes of chromophore separation, reorganization energies, and free energy gaps. It would be possible then to take values of electronic coupling that depend on distance and to solve the model for new parameters appropriate to lower temperatures. This is likely to improve consistency with the experimental results. We. tried to choose parameters in order to obtain results consistent with experiment. It is in this situation necessary to change simultaneously a few parameters.

The changes in the reorganization energies $\lambda$ and electronic couplings $V$ at lower, temperature, which we obtained as a result of fitting the curves, can be explained on the basis of physical arguments, concerning thermal contraction and decrease in the contribution of reorientation components in reorganization energies.

\section{Acknowledgments}

This work was supported in part by the Committee for Scientific Research grant 3 T09 A 09612.

\section{References}

[1] Z.R. Grabowski, K. Rotkiewicz, A. Siemiarczuk, D.J. Cowley, W. Baumann, Nouv. J. Chimie 3, 443 (1979).

[2] K. Rotkiewicz, K.H. Grellmann, Z.R.. Grabowski, Chem. Phys. Lett. 21, 212 (1973).

[3] Z.R. Grabowski, K. Rotkiewicz, W. Rubaszewska, E. Kirkor-Kamińska, Acta Phys. Pol. A 54, 767 (1978).

[4] W. Rettig, W. Majnez, R. Lapouyade, G. Hauke, J. Photochem. Photobiol. A, Chem. 62, 415 (1991).

[5] D.F. Duxbury, Chem. Rev. 93, 381 (1993).

[6] K.Y. Law, Chem. Rev. 92, 449 (1993).

[7] E. Lippert, W. Rettig, V. Bonacic-Kontecky, F. Heisel, J.A. Miehe, Adv. Chem. Phys. 68, 71 (1987).

[8] M.R. Wasielewski, Chem. Rev. 92, 435 (1992).

[9] A.M. Brouver, P.D. Mout, P.H.M. van der Brink, S.A. Jonker, J.M. Warman, Chem. Phys. Lett. 186, 481 (1991). 
[10] R.J. Willemse, J.W. Verhoeven, A.M. Brouver, J. Phys. Chem. 99, 5753 (1995).

[11] W. Schuddeboom, T. Scherer, J.M. Warman, J.W. Verhoeven, J. Phys. Chem. 97, 13092 (1993).

[12] A.M. Brouver, C. Eijkelhoff, R.J. Willemse, J.W. Verhoeven, W. Schuddeboom, J.M. Warman, J. Am. Chem. Soc. 115, 2988 (1993).

[13] R.M. Marcus, Commemorative Issue, J. Phys. Chem. 90, 3657 (1986).

[14] M. Tachiya, J. Phys. Chem.-93, 7050 (1989).

[15] M. Tachiya, J. Phys. Chem. 97, 5911 (1993).

[16] M. Tachiya, Chem. Phys. Lett. 230, 491 (1994).

[17] Y. Hu, S. Mukamel, J. Chem. Phys. 91, 6973 (1989).

[18] Y. Hu, S. Mukamel, in Perspectives in Photosynthesis, The Jerusalem Symposia on Quantum Chemistry and Biochemistry, Vol. 22, Eds. J. Jortner, B. Pullman, Kluwer Academic Publ., Dordrecht 1990, p. 171.

[19] M. Sparpaglione, S. Mukamel, J. Chem. Phys. 91, 3938 (1987).

[20] M. Sparpaglione, S. Mukamel, J. Phys: Chem. 88, 3263 (1988).

[21] I. Rips, J. Jortner, J. Chem. Phys. 87, 6513 (1987).

[22] D. Gust, T.A. Moore, A.L. Moor, Acc. Chem. Res. 26, 198 (1993).

[23] G.J. Small, Chem. Phys. 197, 239 (1995):

[24] M. Bixon, J. Jortner, M.E. Michel-Beyerle, Biochim. Biophys. Acta 301, 1056 (1991).

[25] V. Nagarajan, W.W. Parson, D. Davies, C. Schenck, Biochemistry 32, 12324 (1993).

[26] A. Warschel, W.W. Parson, Ann. Rev. Phys. Chem. 42, 279 (1991).

[27] M. Bixon, J. Jortner, M.E. Michel-Beyerle, Chem. Phys. 197, 389 (1995).

[28] M.E. Michel-Beyerle, G.J. Small, Chem. Phys. 197, 223 (1995).

[29] S. Mukamel, V. Rupasov, Chem. Phys. Lett. 242, 17 (1995).

[30] R. Marcus, Chem. Phys. Lett. 133, 471 (1987).

[31] J.M. Jean, R.A. Friesner, G.R. Flaming, J. Chem. Phys. 96, 5827 (1992).

[32] H. Huber, M. Meyer, T. Naegele, I. Hartl, H. Scheer, W. Zinth, J. Wachtveitl, Chem. Phys. 197, 297 (1995).

[33] D.G. Johnson, M.P. Niemczyk, D.W. Minsek, G.P. Wiederrecht, W.A. Svec, G.L. Gaines III, M.R. Wasielewski, J. Am. Chem. Soc. 115, 5692 (1993).

[34] D. Gust, T.A. Moore, P.A. Liddell, G.A. Nemeth, L.R. Makings, A.L. Moore, D. Barrett, P.J. Pessiki, R.V. Bensasson, M. Rougee, C. Chachaty, F.C. DeSchryver, M. van der Auweraer, A.R. Holzwarth, J.C. Connolly, J. Am. Chem. Soc. 109, 846 (1987).

[35] T.A. Moore, D. Gust, P. Mathis, J.C. Miolocq, C. Chachaty, R.V. Bansasson, E.J. Land, D. Doizi, P.A. Liddell, W.R. Lehman, G.A. Nemeth, A.L. Moore, Nature 307, 630 (1984).

[36] S.-Ch. Hung, S. Lin, A.N. Macpherson, J.M. DeGraziano, P.K. Karrigan, P.A. Liddell, A.L. Moore, T.A.Moore, D. Gust, J. Photochem. Photobiol. A, Chem. 77, 207 (1994). 
[37] A. Osuka, K. Maruyama, N. Mataga, T. Asahi, I. Yamazaki, N. Tamai, J. Am. Chem. Soc. 112, 4958 (1990).

[38] A. Osuka, F. Kobayashi, K. Maruyama, N. Mataga, T. Asahi, T. Okada, I. Yamazaki, Y. Nishimura, Chem. Phys. Lett. 201, 223 (1993).

[39] A. Osuka, T. Nagata, F. Kobayashi, R.P. Zhang, K. Maruyama, N. Mataga, T. Asahi, T. Ohno, K. Nozaki, Chem. Phys. Lett. 199, 302 (1992).

[40] A. Osuka, S. Marumo, K. Maruyama, N. Mataga, Y. Tanaka, S. Taniguchi, T. Okada, I. Yamazaki, N. Nishimura, Bull. Chem. Soc. Jpn. 68, 262 (1995).

[41] J.M. Warman, K.J. Smit, S.A. Jonker, J.W. Verhoeven, J. Kroon, M.N. Paddon-Row, A.M. Oliver, Chem. Phys. 170, 359 (1993).

[42] A. Harriman, F. Odobel, J.P. Sauvage, J. Am. Chem. Soc. 116, 5481 (1994).

[43] J.-P. Collin, A. Harriman, V. Heitz, F. Odobel, J.P. Sauvage, J. Am. Chem. Soc. 116, 5679 (1994).

[44] A. Harriman, V. Heitz, M. Ebersole, H. van Willigen, J. Phys. Chem. 98, 4982 (1994).

[45] J:-C. Chambron, A. Harriman, V. Heitz, J.P. Sauvage, J. Am. Chem. Soc. 115, 6109 (1993).

[46] M. Marchi, J. Gehlen, D. Chandler, M. Newton, J. Am. Chem. Soc. 115, 4178 (1993).

[47] K. Schulten, M. Tesch, Chem. Phys. 158, 421 (1991).

[48] M. Nonella, K. Schulten, J. Phys. Chem. 95, 2059 (1991).

[49] J. Najbar, M. Tachiya, J. Phys. Chem. 98, 199 (1994).

[50] M. Fuschiki, M. Tachiya, J. Phys. Chem. 98, 10762 (1989).

[51] J. Najbar, Bull. Pol. Acad. Sci., Chem. 45, 79 (1997).

[52] J. Najbar, M. Tachiya, J. Photochem. Photobiol. A, Chem. 95, 51 (1996).

[53] T. Motylewski, J. Najbar, M. Tachiya, Chem. Phys. Lett. 212, 193 (1996).

[54] M.H. Cho, R.J. Silbey, J. Chem. Phys. 103, 595 (1995).

[55] R.A. Marcus, Faraday Symp. Chem. Soc. 10, 60 (1975).

[56] M. Tachiya, Chem. Phys. Lett. 23, 491 (1994).

[57] M. Sparpaglione, S. Mukamel, J. Chem. Phys. 60, 2275 (1988).

[58] A. Warschel, Z.T. Chu, W.W. Parson, Science 246, 112 (1989).

[59] L.D. Zusman, Chem. Phys. 41, 295 (1980).

[60] P. Gajdek, Acta Phys. Pol. A 89, 727 (1996).

[61] S. Mukamel, Adv. Chem. Phys. 70, 165 (1988).

[62] W. Zinth, P. Huppmann, T. Arlt, J. Wachtveitl, Philos. Trans. R. Soc. Lond. A 356, 465 (1998).

[63] J.N. Gehlen, M. Marchi, D. Chandler, Science 263, 499 (1994).

[64] J. Tang, J. Norris, J. Chem. Phys. 101, 5615 (1994).

[65] H.A. Murchison, R.G. Alden, J.P. Allen, J.M. Pelloquin, A.K.W. Taguchi, N.W. Woodbury, J.C. Williams, Biochemistry 32, 3498 (1993).

[66] J.C. Williams, R.G. Alden, H.A. Murchison, J.M. Pelloquin, N.W. Woodbury, J.P. Allen, Biochemistry 31, 11029 (1992). 